Recepción: 20 / 04 / 2017

Aceptación: 20 / 05 / 2017

Publicación: 15 / 06 / 2017

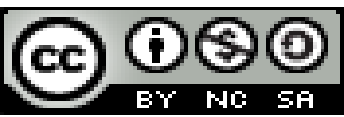

Ciencias Técnicas y Empresariales

Artículo de Investigación

\title{
Afectaciones a integridad de tubería en sección de Oleoducto
}

\section{Affections on pipe integrity in pipeline section}

\section{Afectações integradas de tuberias em seção de Oleoducto}

\author{
Carlos R. Chávez-Romero ${ }^{\mathrm{I}}$ \\ carlosroberto160677@gmail.com \\ Rene A. Carrillo-Flores III \\ rcarrillo@uce.edu.ec \\ Darwin R. Caina-Aysabucha ${ }^{\text {III }}$ \\ drcaina@uce.edu.ec \\ Marcelo P. Carrillo-Flores IV \\ mpcarrillo@siegfried.com.ec \\ Mariana G. Calderón-Defaz V \\ mgcalderon@uce.edu.ec
}

Correspondencia: carlosroberto160677@gmail.com

Ingeniero Mecánico, Andes Petroleum Ecuador Ltd, Universidad Central del Ecuador, Quito, Ecuador.

Magister en Ingeniería Industrial, Msc, Universidad Central del Ecuador. Facultad ee Ingeniería, Ciencias Físicas y Matemática, Quito, Ecuador.

III. Ingeniero en Electrónica Y Telecomunicaciones, Universidad Central del Ecuador. Facultad de Ingeniería, Ciencias Físicas y Matemática, Quito, Ecuador.

IV. Laboratorios Siegfried, Magister en Sistemas de Gestión Integrados, Escuela Politécnica Nacional, Quito, Ecuador.

v. Doctora en Psicología Educativa y Orientación. Especialización Psicología del Adolescente, Universidad Central del Ecuador. Facultad de Ciencias Químicas, Quito, Ecuador. 


\section{Resumen}

Los ductos usados en el transporte de hidrocarburos enfrentan diferentes amenazas a su integridad. Algunas de estas amenazas tienen un origen en factores prevenibles en la etapa de diseño y si no son considerados en esa fase pueden dar origen a afectaciones durante la operación. Un problema repetitivo de arrugamiento en una sección de un oleoducto es abordado con la finalidad de conocer la causa más probable de origen e implementar una solución viable. El problema es enfocado desde un punto de vista de gerencia de proyectos ya que la solución que se implemente debe necesariamente ser aplicable tanto técnica como económicamente pero además oportuna ya que un derrame puede tener consecuencias impredecibles. Los análisis técnicos realizados permiten tener una base sólida para la toma de decisiones que disminuyan los riesgos de un nuevo evento. El documento incluye lecciones aprendidas de manera que se pueda tomar como referencia en la construcción de nuevos ductos y en análisis de problemas similares.

Palabras clave: Ductos; hidrocarburos; diseño; integridad; deformación. 
Carlos R. Chávez-Romero; Rene A. Carrillo-Flores; Darwin R. Caina-Aysabucha; Marcelo P. Carrillo-Flores; Mariana G.

\section{Abstract}

Pipe lines use in the transportation of hydrocarbons are exposed to different threats to its integrity. Some of these threats can be prevented during design stage and if they are not properly considered could produce damages during the operation of the pipeline. A repetitive problem of wrinkles in a pipeline section is studied to determine the most probable cause of it and to implement a solution. The problem is considered from a project management perspective because the solution to be implemented must be affordable technically and economically but also it shall be implemented fast since a spill could drive unpredictable consequences. The technical analysis performed allows having a solid base for decision making with the objective of reducing risks of a new event. The document includes lessons learned so it can be considered as a reference for the construction of new pipe lines and to solve similar problems.

Key words: Pipe line; hydrocarbons; design; integrity; deformation. 


\section{Resumo}

Los ductos foram usados no transporte de hidrocarburos enfrentan diferentes amenazas a su integridad. Algumas de estas amenazas têm um origen em fatores prevenveis no estágio de projeto e se não estão em fase de fase em caso de origem a afectaciones durante a operação. Um problema repetitivo de arrugamento em uma seção de um oleoduto foi abordado com a finalidade de conhecer a causa mais provável de origem e implementar uma solução viável. O problema é enfocado desde o ponto de vista de gestão de projetos, na medida em que é necessário implementar, se necessário, ser aplicável, tanto técnico quanto economicamente, além de oportuna, você pode destruir impredecibles. Los análisis técnicos realizados permitem uma base sólida para o toma de decisões que diminuem os riscos de um novo evento. $\mathrm{O}$ documento inclui lecções aprendidas de moda que podem ser utilizados na construção de novos ductos e análises de produtos similares.

Palavras chave: Ductos; hidrocarburos; diseño; integridade; deformación. 
Carlos R. Chávez-Romero; Rene A. Carrillo-Flores; Darwin R. Caina-Aysabucha; Marcelo P. Carrillo-Flores; Mariana G.

\section{Introducción.}

La construcción de oleoductos en el Ecuador se ha venido realizando por espacio de más de 30 años lo que ha permitido acumular experticia. Por esta razón resulta sorpresivo ver que en muchos casos se considera únicamente como un tema de soldar una serie de tubos y enterrarlos, es decir se considera únicamente una parte de la ingeniería mecánica, y se deja de lado temas importantes como el aseguramiento del flujo, el control de corrosión o la selección del sitio donde irá implantada la tubería.

Los ductos de transporte de hidrocarburos enfrentan una serie de amenazas a su integridad en su operación diaria. Una de las principales tareas de las compañías que producen o transportan crudo o sus productos es proteger la integridad de sus sistemas de tuberías, manteniéndolos aptos para trabajar dentro de sus parámetros de diseño y seguridad. Con esta finalidad, se realizan grandes esfuerzos técnicos y económicos para diseñar e implementar planes de diseño, construcción, inspección y mantenimiento que reduzcan riesgos de deterioro, falla y consecuentes fugas. Pese a este enfoque, existen eventos que inevitablemente, se producen y que deterioran las tuberías ocasionando pérdidas económicas y materiales. La introducción de un oleoducto y la construcción de su respectivo derecho de vía son alteraciones al entorno que se encuentra en equilibrio, por lo que es importante considerar potenciales consecuencias.

A lo largo de los años las operadoras han acumulado conocimiento en la gestión de riesgos y han colectado información que les permite lidiar de manera eficiente con las amenazas que puedan desencadenar problemas en las tuberías. Parte de ese conocimiento son los datos históricos de inspecciones y eventos producidos, mismos que permiten establecer estrategias de mitigación para evitar que se repitan los incidentes y mejore la gestión. Esta información es ordenada y analizada de 
manera crítica conforme a prácticas aceptadas en la industria [5] [6], de manera que permita tomar las mejores decisiones, técnicas y económicas, para mantener el ducto operativo. En otras palabras, las decisiones deben ser tomadas a tiempo, deben ser aplicables a la realidad de la operación local y del recurso disponible y, algo fundamental en la industria, deben tener el menor costo posible.

La investigación analiza un proceso de fallas recurrentes en un área puntual del derecho de vía de un oleoducto principal de transporte de crudo con el enfoque de proporcionar una solución viable y oportuna que permita a la línea continuar trabajando con seguridad en corto tiempo, minimizando costos relacionados a la implementación de la solución y a pérdidas de producción. Se aborda además a través del ejemplo como el pasar por alto en la etapa de diseño temas relacionados a operación o mantenimiento de la línea, pueden desencadenar o enmascarar procesos importantes de afectación a los ductos de transporte de fluido. El enfoque planteado es de gerencia de proyectos, que busca solucionar el problema, y no busca un enfoque de análisis de falla. Se pretende además que el presente documento sea una fuente de información para quienes requieren construir ductos o enfrentar problemas similares.

Bajo estas consideraciones, se realiza una investigación para establecer la causa o causas de eventos repetitivos de afectación a una sección de un oleoducto con la finalidad de poner sobre la mesa posibles soluciones aplicables técnica y económicamente y seleccionar e implementar la más viable. Una descripción de los eventos producidos hasta el momento y los resultados de las inspecciones tanto en dichos eventos como realizados posteriormente, son la base para establecer la estrategia que permita identificar la causa del problema y una vez conocida esta, analizar soluciones probables. Las características del oleoducto se presentan en la siguiente tabla. 
Carlos R. Chávez-Romero; Rene A. Carrillo-Flores; Darwin R. Caina-Aysabucha; Marcelo P. Carrillo-Flores; Mariana G.

\begin{tabular}{|c|c|c|c|c|c|}
\hline $\begin{array}{c}\text { Diámetro } \\
\text { nominal } \\
\text { (pulgadas). }\end{array}$ & Longitud (m) & Material & $\begin{array}{c}\text { Espesor nominal } \\
\text { (pulgadas) }\end{array}$ & Fluido & Año construcción \\
\hline 12 & 72500 & API 5L X52 & 0.250 & Crudo & 1999 \\
\hline
\end{tabular}

Tabla $N^{\circ}$ 1.- Datos del ducto [11].

\section{Descripción de eventos acontecidos.}

En diciembre del año 2006 (ver figura 1) se presenta un evento de pérdida de contención del ducto en el área comprendida del kilómetro 20+227 del derecho de vía del oleoducto en estudio. Las fotos disponibles indican la presencia de una arruga en la tubería en el sitio donde se produjo el incidente [11].

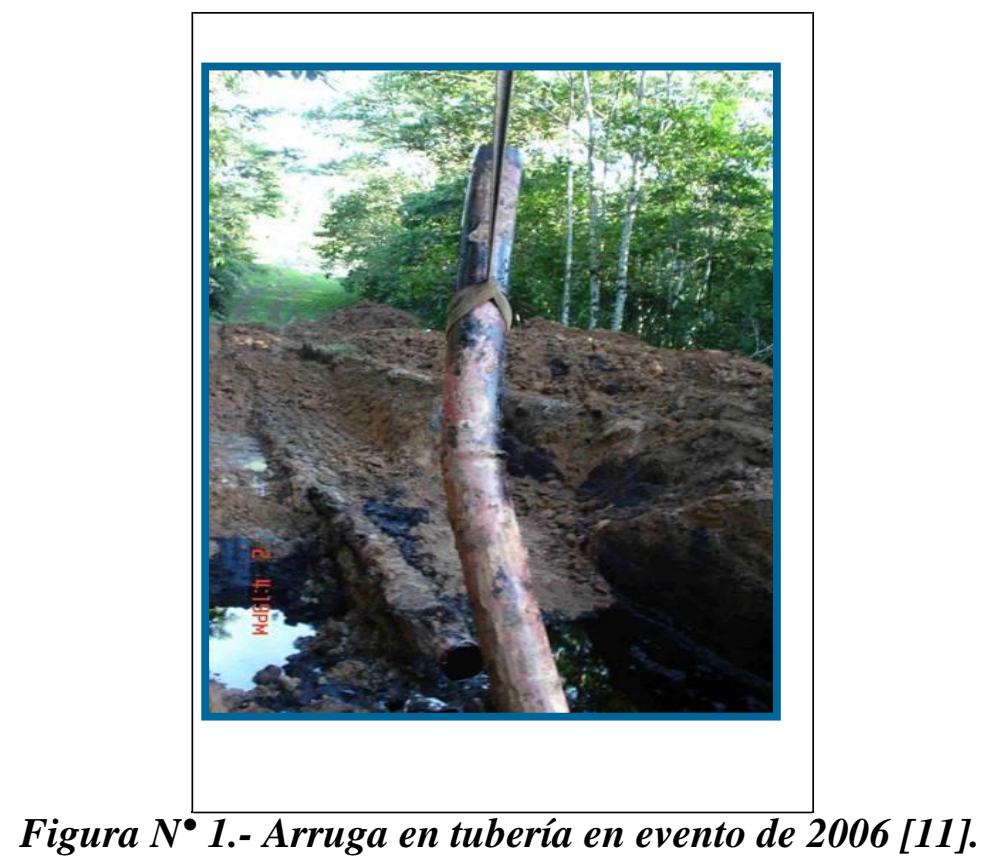

En mayo del año 2007 se produce un nuevo evento en el área del kilómetro 19+780. En esta ocasión se encuentran dos secciones arrugadas del tubo de acuerdo a las evidencias disponibles [11] en la figura 2. 


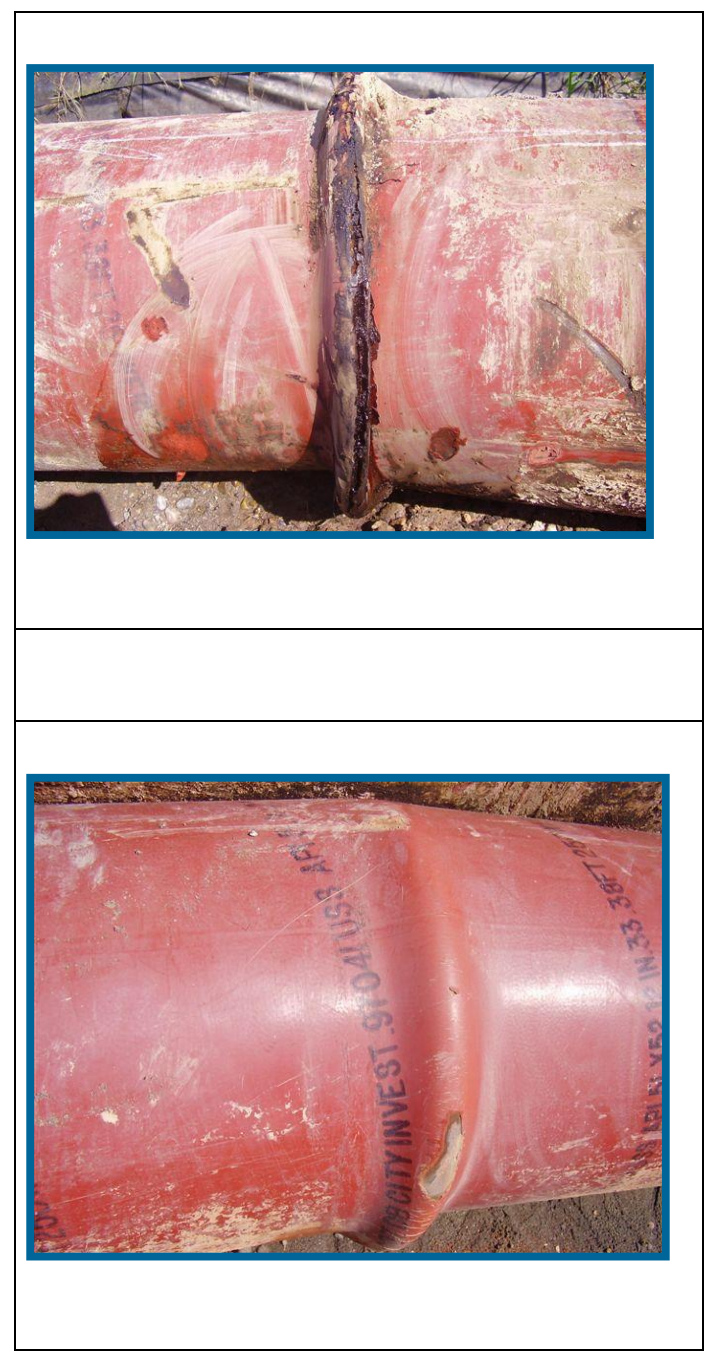

Figura $N^{\circ}$ 2.- Arrugas en tuberías en evento de 2007 [11].

En el año 2014 se produce otro evento en la misma área, a la altura del kilómetro 21, encontrándose nuevamente una arruga en el sitio de deterioro. En esta ocasión es factible realizar una inspección detallada al sitio en que se produce la afectación, encontrándose además evidencias de deterioro en el área circundante y en la carretera cercana causadas por hundimientos [12], esto se puede apreciar en la figura 3. 
Carlos R. Chávez-Romero; Rene A. Carrillo-Flores; Darwin R. Caina-Aysabucha; Marcelo P. Carrillo-Flores; Mariana G. Calderón-Defaz

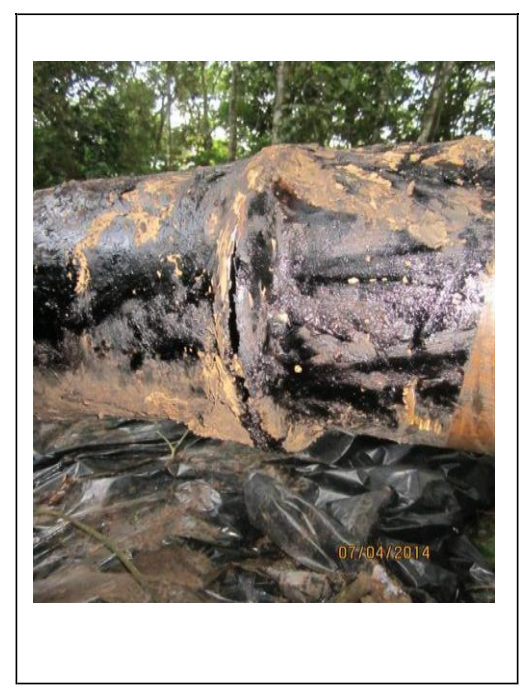

Figura $N^{\circ}$ 3.- Arruga en tubería en evento de 2014 [12].

La figura 4 muestra la localización de los eventos, el derecho de vía y la carretera en un plano.

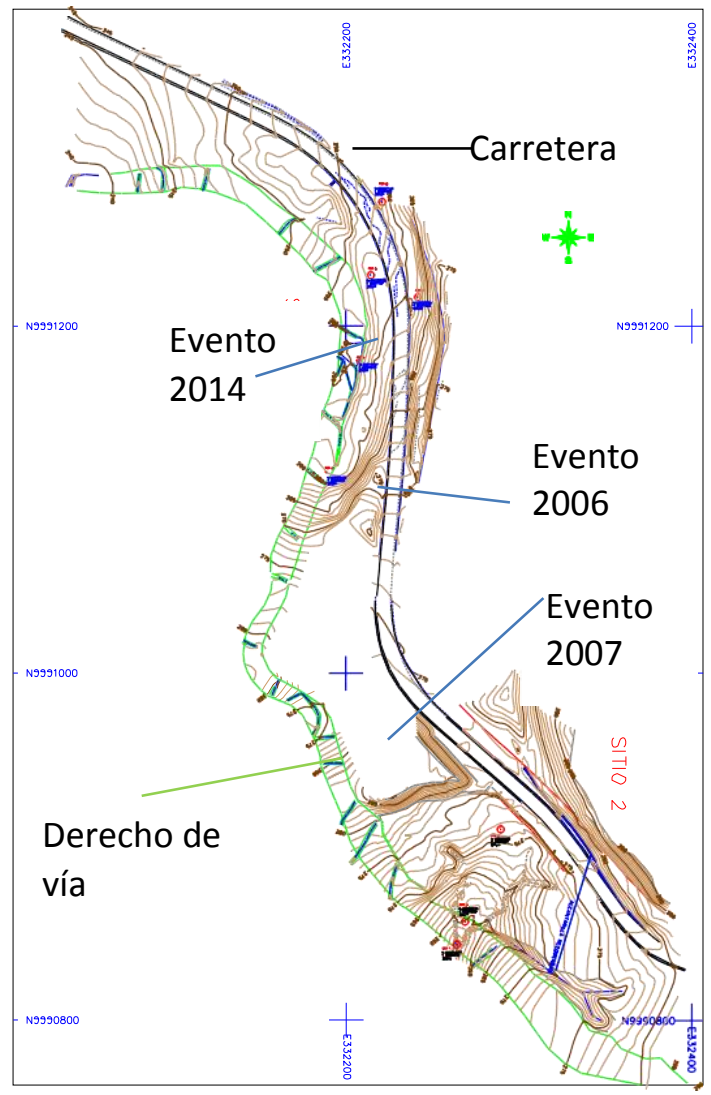

Figura 4.- Ubicación de eventos en plano. 


\section{Materiales y métodos.}

\section{Procedimiento}

Inspección y análisis de los eventos desde el punto de vista Mecánico.

Con la finalidad de verificar la existencia deformaciones y pérdida de espesor ocasionado por corrosión a lo largo de la extensión total de la tubería, se corren dos herramientas instrumentadas. Las tecnologías usadas son de sensores de detección de deformaciones (DEF) para detectar y dimensionar arrugamientos y deformaciones y de fuga de flujo magnético axial (MFL) para ubicación y dimensionamiento de corrosión interna y externa.

La inspección con herramienta DEF reporta deformaciones en diferentes ubicaciones de la tubería. [13]. Se realizan validaciones (inspecciones en sitio) para verificar la validez de los datos entregados por las herramientas de inspección y verificar las anomalías reportadas. En el km 63+382 se tienen 3 anomalías muy cercanas lo que puede ser indicio de existencia de una arruga. La verificación en sitio descarta la presencia de arrugas, sin embargo, se encuentran defectos relacionados a daños con maquinaria pesada y la tubería es reparada. De acuerdo a ASME B31.4, una deformación (dent) debe ser reparada cuando presenta pérdida de material y una deformación de más del 6\% del diámetro nominal [9]. 
Carlos R. Chávez-Romero; Rene A. Carrillo-Flores; Darwin R. Caina-Aysabucha; Marcelo P. Carrillo-Flores; Mariana G.

Calderón-Defaz

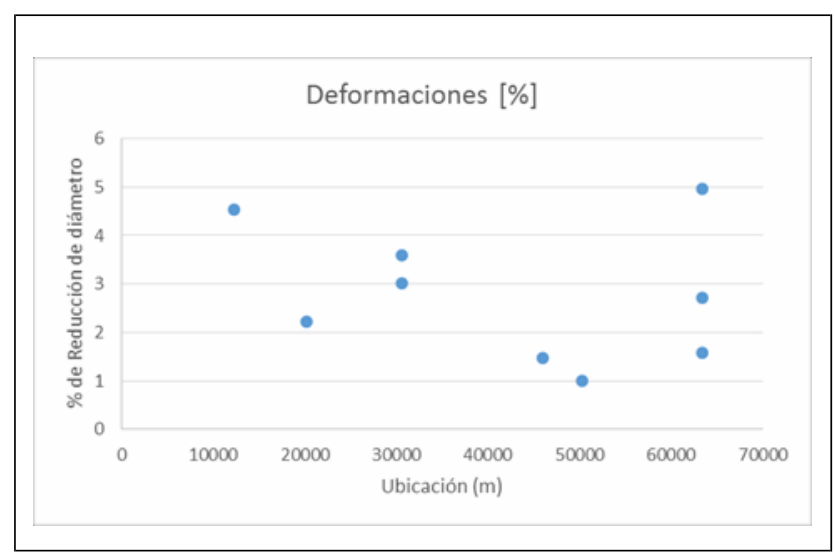

Figura $N^{\circ}$ 5.- Ubicación y profundidad de deformaciones detectadas con DEF, en base a los datos de [13].

Como se puede ver, al momento de la inspección no existen deformaciones importantes en el área de estudio. No se tiene evidencia en esta inspección, ni históricamente en inspecciones pasadas, de presencia de arrugamientos de la tubería en otros sectores distintos al área de los kilómetros 19$21[13]$.

Se analizan los resultados de la inspección con la herramienta MFL a fin de detectar disminuciones de espesores que pueden dar indicios de afectación a la tubería. Se encuentran disminuciones menores que no representan un riesgo a la tubería y no se encuentran evidencias de pérdidas de espesor internas o externas en la tubería a la altura del kilómetro 19 al 21[13]. Es importante considerar que la tolerancia de detección de la herramienta es $10 \%$ del espesor nominal por lo que algunas indicaciones pueden ser errores de medición [13]. 
Afectaciones a integridad de tubería en sección de Oleoducto

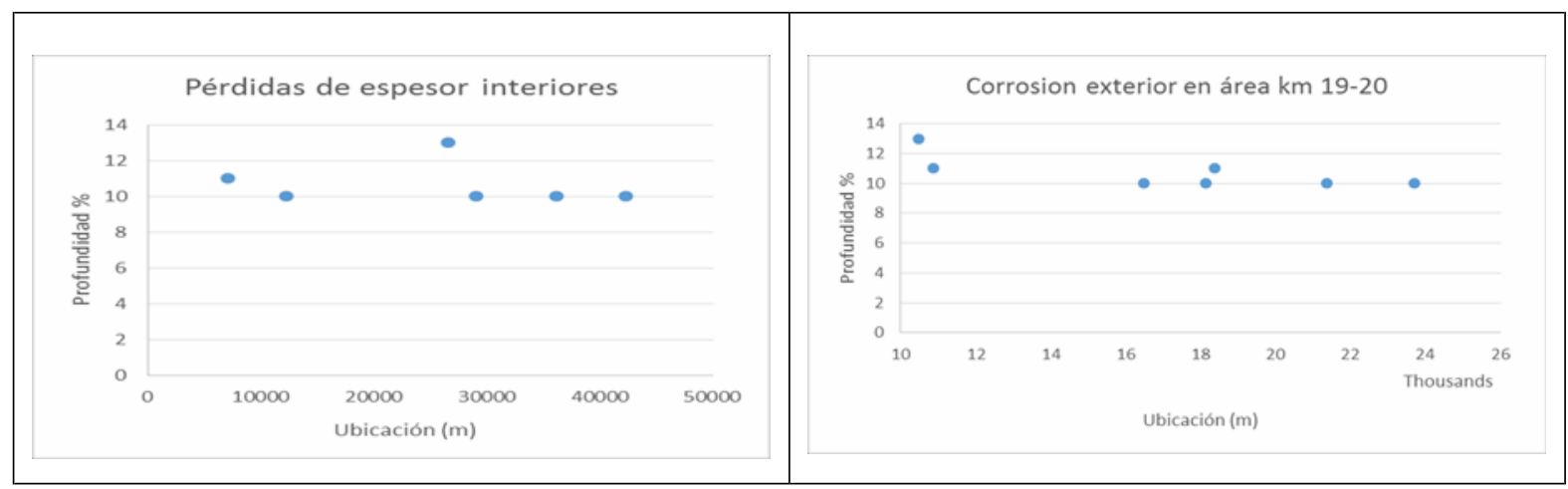

Figura $N^{\circ}$ 6.- Ubicación y profundidad de pérdidas de espesor interiores y exteriores detectadas con MFL en los kilómetros 19-21, en base a los datos de [13]

La herramienta muestra además datos de espesores de la línea con variaciones que van desde los 7 hasta los 17 milímetros (ver figura 7). El tener espesores variables a lo largo de una tubería imposibilita la detección de deformaciones como abolladuras y arrugas a través de una metodología simple, efectiva y de bajo costo como lo es la inspección con placa calibradora.

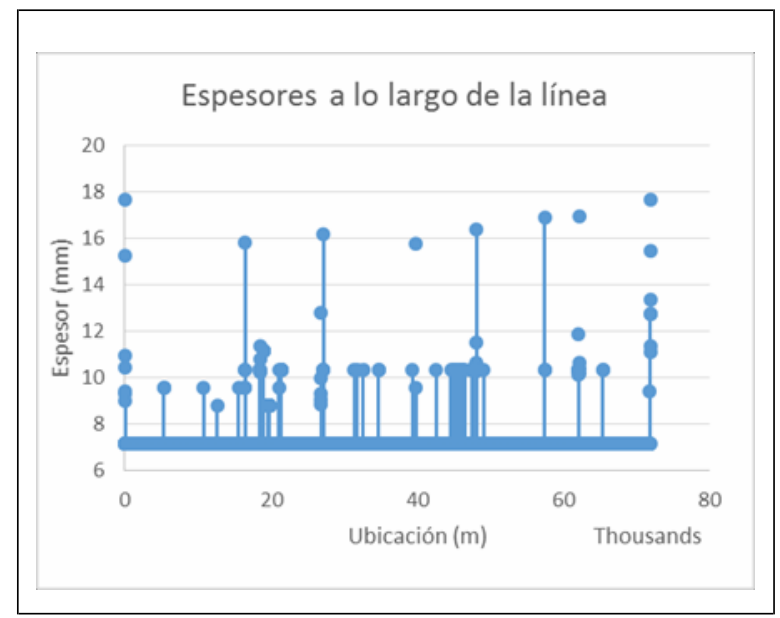

Figura 7: Espesores de la tubería a lo largo de la línea, en base a los datos de [13].

Al no existir evidencia, histórica ni actual, de arrugamientos fuera del área comprendida entre el kilómetro 19 y 21 del derecho de vía del oleoducto objeto de estudio y con los resultados de las inspecciones con herramientas instrumentadas, sumado a la consulta de varias publicaciones que consideran la formación de arrugas en tuberías [1,4,13] puede concluirse que los arrugamientos en 
Carlos R. Chávez-Romero; Rene A. Carrillo-Flores; Darwin R. Caina-Aysabucha; Marcelo P. Carrillo-Flores; Mariana G.

el presente caso no se presentan por temas relacionados a la idoneidad de la tubería. La operación continua desde el año 1999 sin problemas de arrugamientos a lo largo de los 72.5 kilómetros, salvo en el sitio puntual de estudio, refuerza esta conclusión.

El tipo de falla observado (arrugas) corresponde a la afectación que se produce por empuje del suelo en las direcciones longitudinal y transversal, mismas que llevan a la tubería más allá de su tensión máxima de fluencia, deformándola. [13] La magnitud y severidad de una arruga puede incrementarse con el tiempo, pudiendo causar problemas de fugas en la tubería [1]. En la figura 8, se muestra un estado de carga que se considera para la simulación de la interacción suelo- tubería usada para el análisis de arrugas a través de modelado de elementos finitos [4] siendo esta la metodología preferida para estudiar y monitorear las arrugas. En el caso presente, no es el objetivo estudiar la arruga ni monitorearla ya que la fuga se ha producido, sino hallar una solución para eliminar este problema recurrente. Por tanto, un modelo de elementos finitos no aplica para los propósitos de estudio de arrugamiento.

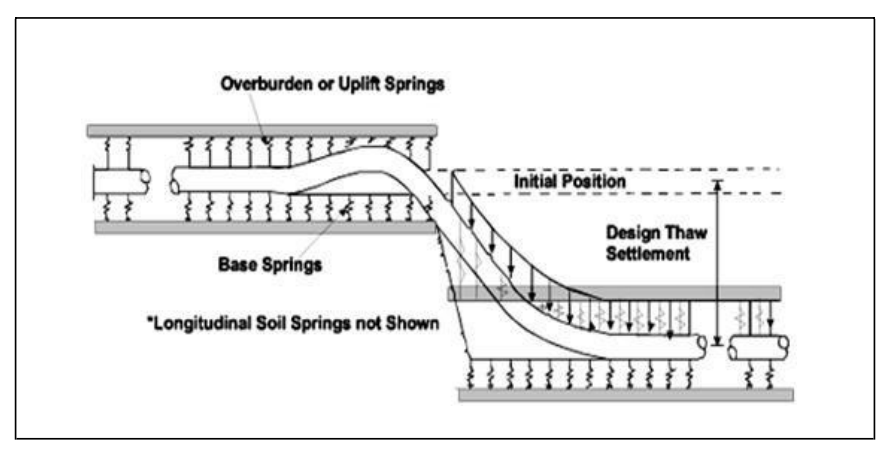

Figura $N^{\circ}$ 8.- Modelo clásico para interacción suelo- ducto [4].

Análisis desde el punto de vista Geológico y Geotécnico.

Se realiza una evaluación geológica y geotécnica de los sitios en que se produjeron los eventos y sus alrededores con la finalidad de encontrar evidencias de la causa de los arrugamientos. 
La inspección visual permite evidenciar claramente que el suelo del área en que se han producido los eventos se encuentra sometido a un proceso de inestabilidad al ser evidentes hundimientos en la carretera próxima, movimientos de suelos, cuarteamientos, etc. Un resumen de los resultados de los análisis practicados y los principales hallazgos se presenta en las siguientes páginas de manera gráfica.

Se determina que existen tres factores que desencadenan la situación de inestabilidad:

Primer factor. - El cambio del uso del suelo, al reemplazarse la vegetación selvática original por pastos, sumado al pastoreo bobino y caballar, produce una alteración de la escorrentía de las aguas lluvias, las que no llegan en su totalidad (existe empozamiento) ni con la misma velocidad a los cauces naturales en los que depositan sus aguas [2]. Producto de esta alteración, gran cantidad de la escorrentía queda retenida en las laderas de los potreros, por la existencia de las huellas dejadas por las pesuñas del ganado. El agua se empoza y penetra en el suelo, cambiando las características mecánicas de los suelos superficiales y de aquellos no muy profundos, creando planos de falla al llegar a contactos de materiales más resistentes y provocando deslizamientos de diversas magnitudes [2]. Este hecho se presenta a lo largo de toda el área de estudio, entre los kilómetros 19 y 21 del derecho de vía, esto se puede apreciar en la figura 9. 
Carlos R. Chávez-Romero; Rene A. Carrillo-Flores; Darwin R. Caina-Aysabucha; Marcelo P. Carrillo-Flores; Mariana G.

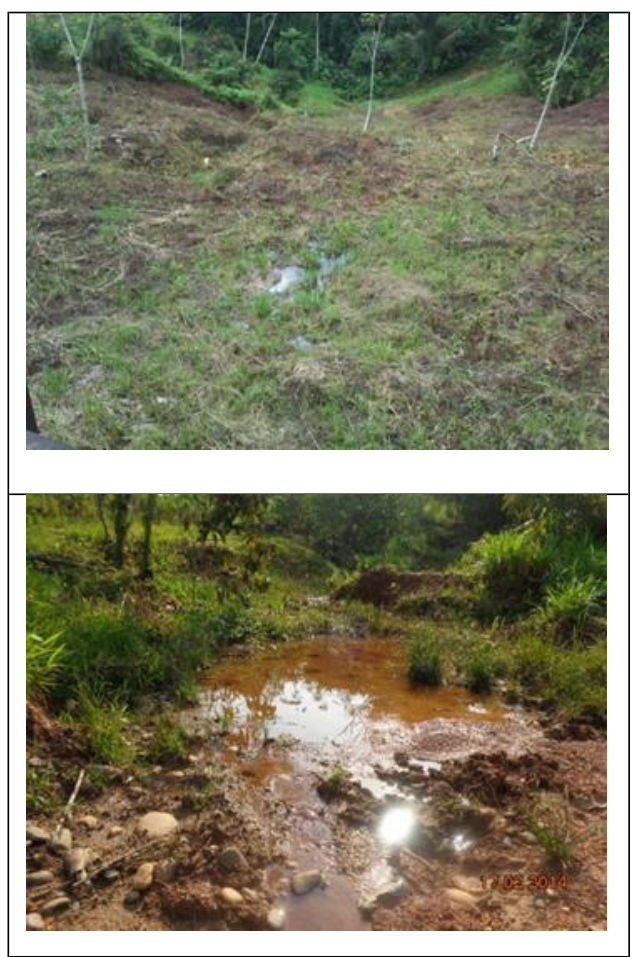

Figura $N^{\circ}$ 9.- Condiciones del suelo alteradas. Fotos tomadas en derecho de vía de oleoducto en estudio.

Segundo factor. - El método de construcción de carreteras a media ladera, como en el caso presente, implica el desalojo del material de corte sobre el talud existente. Si la pendiente transversal es mayor al 45\%, que es la pendiente máxima para admitir relleno de acuerdo a las prácticas aceptadas de ingeniería, se crea una zona de riesgo de deslizamientos [2]. Mediante un levantamiento topográfico se pudo comprobar que una parte importante del derecho de vía en estudio ha sido construida sobre escombreras inestables con gradientes cercanas al $80 \%$.

Las perforaciones y ensayos de laboratorio efectuados dentro del análisis geotécnico muestran evidencia de que la tubería fue enterrada posiblemente en relleno producto de los desechos de la construcción de la vía aledaña. 
Ensayos SPT (standard penetration test) (ASTM D 1586), son practicados en diferentes sitios del área de estudio con la finalidad de evaluar la resistencia a la penetración del suelo. El punto P4 como se ve en la figura 10, se encuentra en el área en que se produjo el evento de 2014. Puede observarse que, hasta una profundidad de 8 metros el número de golpes requerido para penetrar es de máximo 10, lo que indica un suelo de consistencia comparativamente blanda. La descripción del suelo es de arcillas y limos de alta compresibilidad en su mayoría [2].

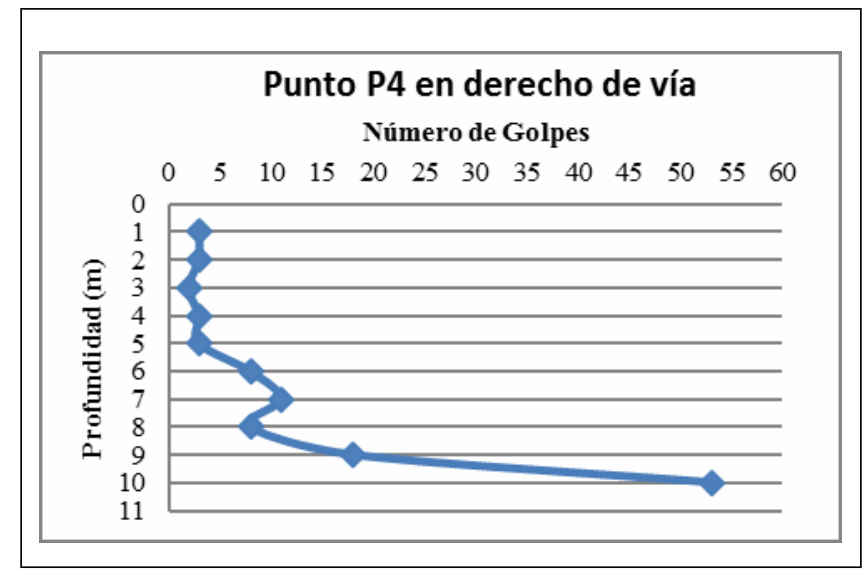

Figura 10.- Resultados de SPT en área de evento de 2014. Autoría personal en base a los datos de [2]

Los puntos P6’y P7’fueron tomados cerca de la carretera, también en el área del evento de 2014. En la figura 11, se puede observarse una estructura comparativamente blanda hasta aproximadamente los 5 metros. 
Carlos R. Chávez-Romero; Rene A. Carrillo-Flores; Darwin R. Caina-Aysabucha; Marcelo P. Carrillo-Flores; Mariana G.

\section{Calderón-Defaz}
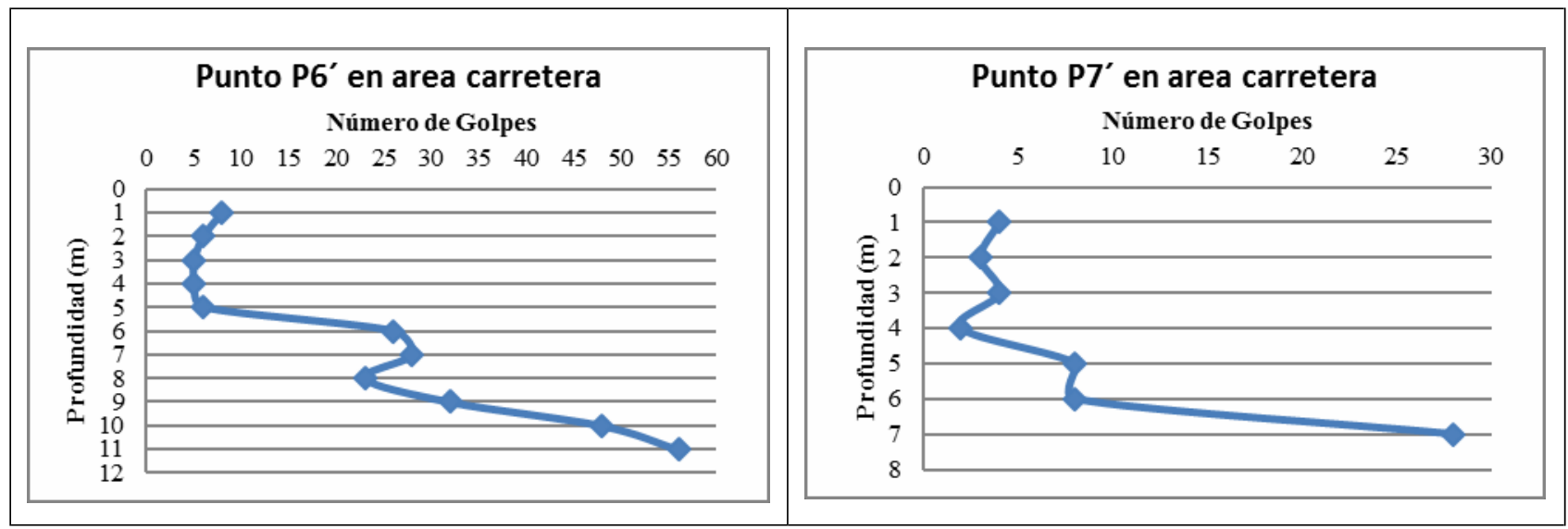

Figura $N^{\circ}$ 11.- Resultados de SPT en área de carretera de evento 2014. Autoría personal en base a los datos de [2].

Con los resultados de los ensayos antes mencionados, se realiza una simulación de estabilidad, en la cual es posible apreciar de manera clara, las secciones comparativamente blandas del suelo, lo que unido al perfil y las evidencias de las muestras inalteradas de campo muestra que el suelo presenta tendencias de inestabilidad hasta profundidades de más de 5 metros. Es importante mencionar que los oleoductos se encuentran enterrados normalmente a una profundidad de 1.2 metros que excede las recomendaciones técnicas de 0.9 metros de la normativa de construcción y gestión de riesgos de ductos [6,9], esto se puede apreciar en la figura 12.

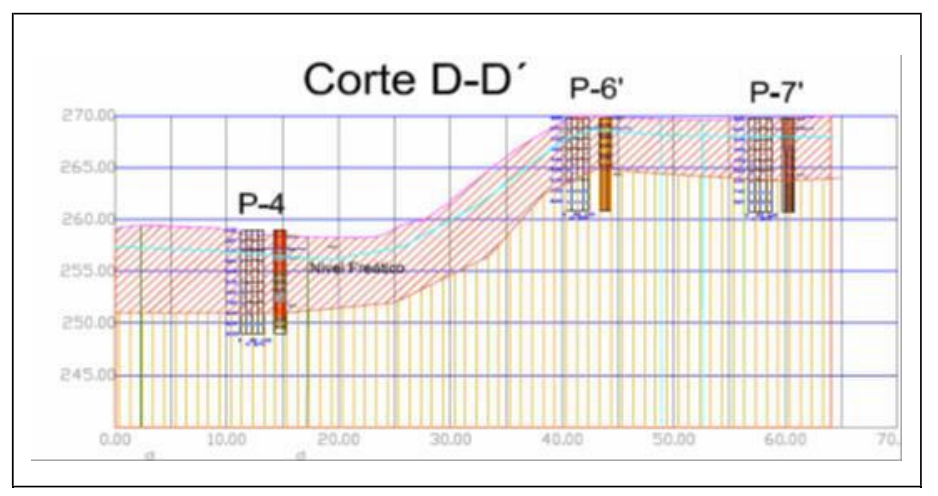

Figura 12: Análisis de estabilidad. [2] 
Ensayos similares se realizan en las otras áreas donde han ocurrido eventos, mostrándose tendencias similares en cuanto a la estabilidad del terreno.

Tercer factor. - Las deficiencias constructivas de la carretera aledaña al derecho de vía en estudio, especialmente en lo referente al sistema de drenaje han contribuido a la inestabilidad del sitio. Cunetas que no funcionan o rotas, sin desfogue a canales construidos técnicamente, producen que el agua no sea desalojada y se infiltre en el suelo, saturándolo. La figura 13 describe gráficamente los aspectos mencionados.

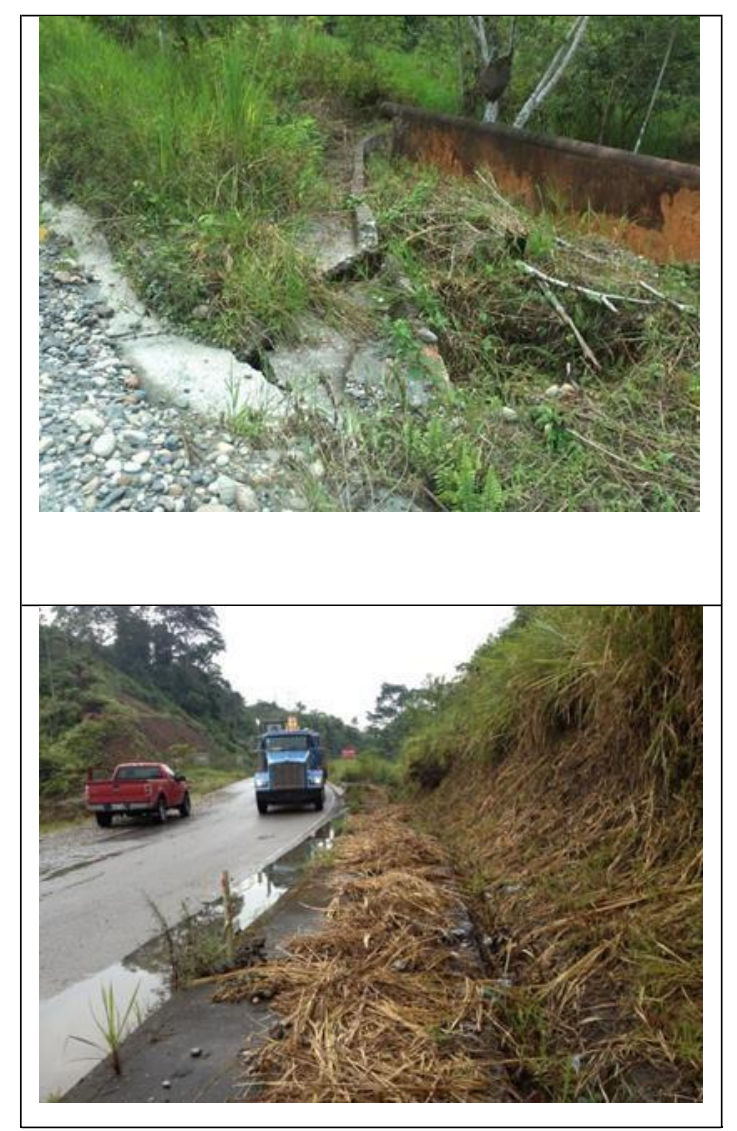

Figura $N^{\circ}$ 13.- Cunetas rotas y que no funcionan. Fotos tomadas en vía ubicada junto al derecho de vía en estudio. 
Carlos R. Chávez-Romero; Rene A. Carrillo-Flores; Darwin R. Caina-Aysabucha; Marcelo P. Carrillo-Flores; Mariana G.

Se hace evidente además el desplazamiento o hundimiento de los suelos en el área al realizar inspecciones en la carretera, tal como se muestra en la figura 14.

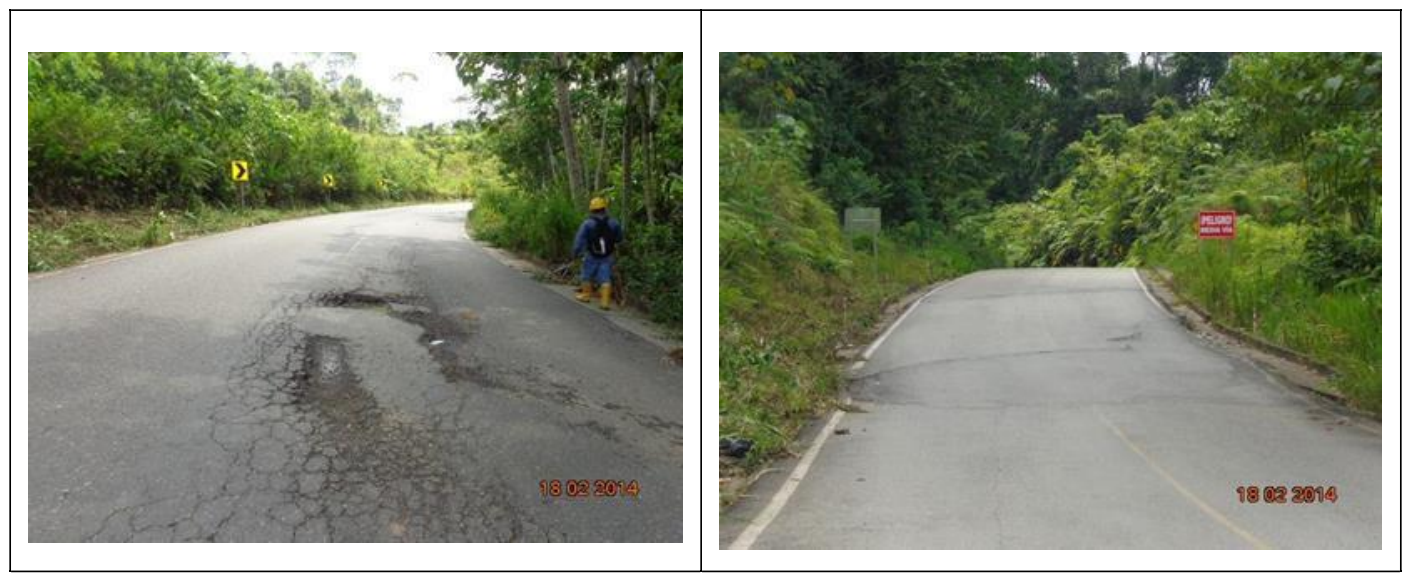

Figura $N^{\circ}$ 14.- Evidencias de hundimientos y desplazamientos del suelo [2].

Con base en los análisis realizados, se concluye que las fallas del ducto se producen el empuje causado por movimientos de tierra inducidos por acumulación y filtración de agua en la parte superior de los taludes y la consiguiente saturación de los materiales que lo conforman. Aunado a que el derecho de vía del oleoducto se encuentra sobre una zona de escombreras producto de la ampliación de la carretera y al drenaje deficiente de la vía que contribuye a la saturación de los suelos del área. [2]

El planteamiento de soluciones debe considerar que la inestabilidad no se produce en una zona puntual sino en toda un área que abarca el sector de los 3 kilómetros estudiados en este documento.

\section{Planteamiento de soluciones.}


Los resultados de los análisis realizados permiten establecer dos escenarios para solucionar este problema:

\section{Primer escenario: Estabilizar el área.}

El alcance de esta solución contempla:

Reconstruir la mesa de la carretera en una longitud de aproximadamente 800 metros y el sistema de drenaje de la misma para que las aguas escurran por las estructuras previstas, sin comprometer la estabilidad del sector.

Construcción de sub-drenes a lo largo de la vía para recolectar las aguas y encausarlas debidamente.

Realizar una campaña de concientización con los vecinos del derecho de vía de manera que se controle la deforestación y evaluar la posibilidad de evitar el pastoreo en áreas que puedan comprometer el derecho de vía.

Excavación controlada a lo largo de los kilómetros 19 a 21 del derecho de vía de manera que el oleoducto se libere de los esfuerzos inducidos por los movimientos de suelos que se hayan dado hasta el momento. Reemplazo de suelos saturados por suelos secos.

Instalación de un sistema de monitoreo de movimiento de suelos en base a mojones superficiales, inclinómetros y sensores de variación de esfuerzos (strain gages).

\section{Considerando los factores siguientes:}

Poca probabilidad de controlar el cambio de uso del suelo en las partes altas del derecho de vía, lo que somete a riesgo de deslizamiento a la línea. 
Carlos R. Chávez-Romero; Rene A. Carrillo-Flores; Darwin R. Caina-Aysabucha; Marcelo P. Carrillo-Flores; Mariana G.

Nulo mantenimiento de la vía y pocas expectativas de que el gobierno decida arreglarla ya que por ser un bien público el estado debe hacerlo y no hay posibilidad de que se lo realice de manera privada.

Esta solución es descartada.

Segundo escenario: Construir una variante al trazado actual del oleoducto.

El alcance de esta solución contempla:

Realizar estudios de cartografía para establecer rutas probables del derecho de vía.

Ensayos geotécnicos para determinar condiciones del suelo en el área seleccionada.

Calculo de cantidades de obra y tubería.

Construcción de derecho de vía y oleoducto de la variante.

Plan de mantenimiento.

Esta opción requiere encontrar un área estable, a largo plazo, a donde llevar la tubería, y que se encuentre lo suficientemente cerca para ser aceptable económicamente.

Considerando que esta opción es técnicamente aplicable y que elimina la dependencia de entidades gubernamentales para la estabilidad del área, se aplica esta opción.

\section{Implementación de la solución.}

La ruta seleccionada para el derecho de vía de la variante debe permitir la construcción de la sección del oleoducto garantizando una operación segura de la línea de transporte de fluido en la sección objeto de estudio. Problemas como el actual se presentan en buena parte debido a la sub estimación de los factores geológicos y geotécnicos en la integridad del oleoducto. Es primordial el enfoque multidisciplinario para encontrar las mejores alternativas. 
Siguiendo los pasos descritos en el punto anterior, se presentan los resultados a lo largo de las siguientes páginas.

\section{Selección de ruta de la variante.}

Con la finalidad de elegir la mejor ruta disponible para construir la variante del oleoducto que evite el tramo inestable de los kilómetros 19 - 21 del derecho de vía existente, se realizó un estudio de selección de derecho de vía, mismo que involucra diferentes actividades y áreas de conocimiento (ver figura 15). Los pasos seguidos son los siguientes:

\section{Estudio en Cartas Topográficas}

Restitución de la faja requerida para el estudio de la variante

Trazado sobre restitución de todas las rutas posibles

Recorrido de campo de las rutas por especialistas.

Evaluación de posibles problemas que puedan presentarse en el derecho de vía y posibles soluciones.

Selección de la ruta más conveniente.

Como paso inicial se determinan diferentes opciones de rutas posibles, a través de el estudio de cartas y restitución aerofotogramétrica. Las opciones determinadas inicialmente se muestran en la figura 20, identificadas por números y colores.

Cada una de estas secciones es recorrida por un equipo de especialistas Geólogos, Geotectónicos, Hidráulicos y Viales de manera que se pueda realizar una evaluación 
Carlos R. Chávez-Romero; Rene A. Carrillo-Flores; Darwin R. Caina-Aysabucha; Marcelo P. Carrillo-Flores; Mariana G.

multidisciplinaria del terreno y se disminuyan las posibilidades de pasar por alto indicaciones importantes.

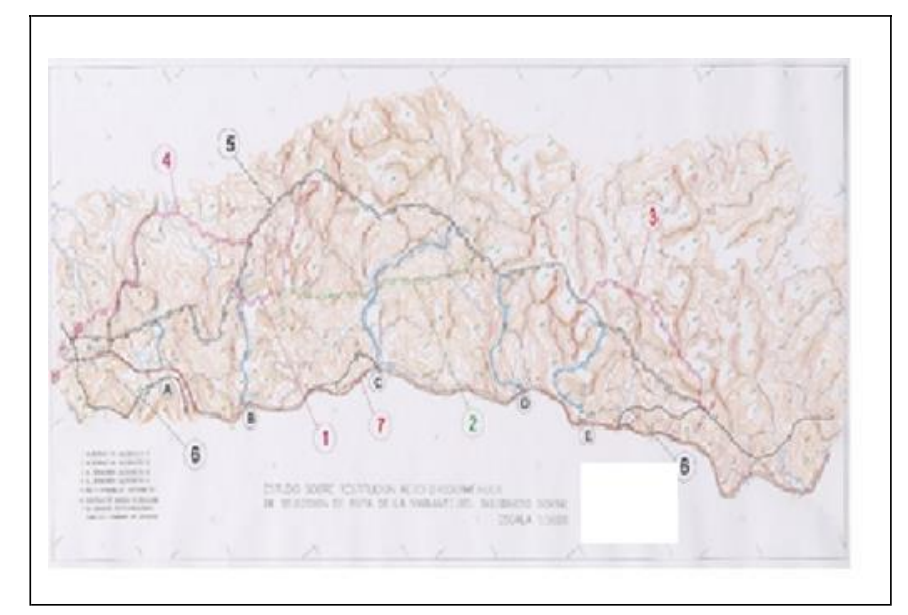

Figura $N^{\circ}$ 15.- Alternativas de rutas. [3]

Como producto de los recorridos se escoge la mejor ruta posible considerando los análisis del grupo de especialistas mencionado anteriormente [3]. Se presenta el resultado de la selección con la ruta replanteada en campo, haciéndose notar que es una combinación de algunas de las rutas propuestas lo cual resalta la importancia de la verificación en campo de las alternativas planteadas en trabajos de oficina, esta selección se puede ver en la figura 16.

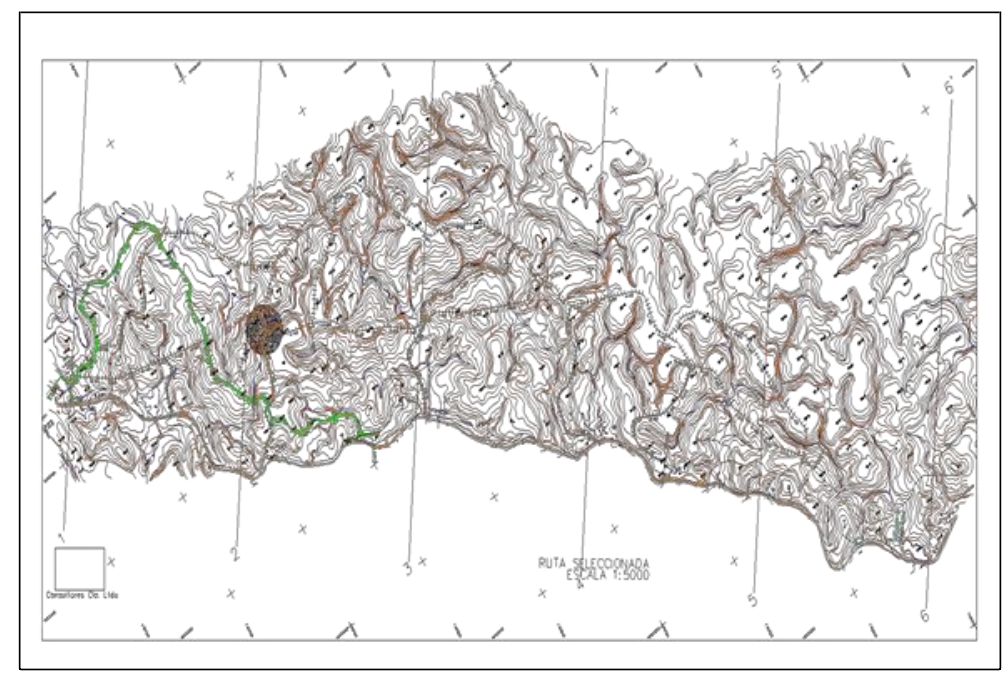

Figura $N^{\circ}$ 16.- Ruta seleccionada [3] 
La inspección de campo acorta la longitud de la variante al determinarse que secciones aguas arriba de la propuesta en las que el ducto se encuentra instalado son estables.

\section{Ensayos geotécnicos.}

Se realizan perforaciones y excavaciones en diferentes puntos a lo largo del derecho de vía con la finalidad de tomar muestras alteradas e inalteradas que servirán para hacer análisis de taludes y estabilidad.

En términos generales los suelos son predominantemente arcillas y limos con contenidos que varían de grava y arena. Estos suelos son muy sensibles al contenido de agua siendo esta una de las razones por las que se buscó que, en lo posible, el derecho de vía se encuentre en las partes altas de las elevaciones del terreno.

Se calcula la capacidad de carga admisible en los diferentes puntos del suelo como un factor que permite conocer la resistencia del suelo y la estabilidad del sector. Se tienen los siguientes resultados [3]:

\begin{tabular}{|c|c|c|c|c|c|c|c|}
\hline $\begin{array}{l}\text { Prof. } \\
\text { (m) }\end{array}$ & $\begin{array}{c}P-1 \\
q_{a}\left(t / m^{2}\right)\end{array}$ & $\begin{array}{c}\mathrm{P}-2 \\
\mathrm{q}_{a}\left(t / \mathrm{m}^{2}\right)\end{array}$ & $\begin{array}{c}\text { P-3 } \\
q_{a}\left(t / m^{2}\right)\end{array}$ & $\begin{array}{c}P-4 \\
q_{=}\left(t / m^{2}\right)\end{array}$ & $\begin{array}{c}\text { P.5 } \\
q_{a}\left(t / m^{2}\right)\end{array}$ & $\begin{array}{c}\mathrm{P}-6 \\
\mathrm{q}_{\mathrm{a}}\left(\mathrm{t} / \mathrm{m}^{2}\right)\end{array}$ & $\begin{array}{c}\text { P-7 } \\
q_{0}\left(t / m^{2}\right)\end{array}$ \\
\hline 0.00 & 10.17 & 5.08 & 6.78 & 11,86 & 5.08 & 6.78 & 9.49 \\
\hline 1.00 & 19.30 & 3.46 & 23.01 & 23,85 & 11.58 & 9.65 & 15.44 \\
\hline 2.00 & 99.68 & 3.32 & 26.38 & 31.18 & 19.91 & 13.12 & 95.36 \\
\hline 3.00 & 85.74 & 3.09 & 34.30 & 65.48 & 16.59 & 13.89 & 135.12 \\
\hline 4.00 & 132.62 & 3.06 & 136.47 & 135.76 & 21.88 & 13.74 & 135.06 \\
\hline 5.00 & 129.42 & 21.16 & 130.01 & 132.74 & 19.02 & 13.69 & 131.07 \\
\hline 6.00 & 132.65 & 21.89 & & 136.84 & 16.76 & 18.15 & 132.65 \\
\hline 7.00 & & 21.60 & & & 31.38 & 20.68 & 9.49 \\
\hline 8.00 & & 22.98 & & & 33.86 & 29.02 & 15.44 \\
\hline
\end{tabular}

Tabla $N^{\circ}$ 2.- Capacidades de carga del suelo [3]. 
Carlos R. Chávez-Romero; Rene A. Carrillo-Flores; Darwin R. Caina-Aysabucha; Marcelo P. Carrillo-Flores; Mariana G.

Como referencia una regla de dedo de los ingenieros civiles es que el suelo tenga al menos una capacidad portante de 15 ton/ m2 para la construcción de una casa. Esto se consigue en el presente caso a profundidades de entre 1 y 2 metros lo que indica que se tiene una capacidad que indica una estabilidad adecuada para el oleoducto. Como se mencionó anteriormente el agua es un factor crítico por lo que las obras de control de recolección y desfogue son prioritarias para el mantenimiento. Puntos que presenten valores de carga por debajo de lo esperado serán evaluados para implementar obras de mitigación, en caso de requerirse.

Al ser el Ecuador un país con historial sísmico, se considera el factor sismo en la estabilidad de 3 secciones del derecho de vía identificadas como propensas a fallas durante la inspección en sitio. Los valores para la determinación de los factores de sismo se toman de la norma NEC "Norma Ecuatoriana de la Construcción (NEC) 2011”. La figura 17, tomada de la norma mencionada permite evidenciar que mayoritariamente la zona petrolera norte ecuatoriana, donde se ubica el ducto objeto de estudio, se encuentra ubicada en una sección con una aceleración sísmica de 0.15 , la más baja de la escala de la norma [8].

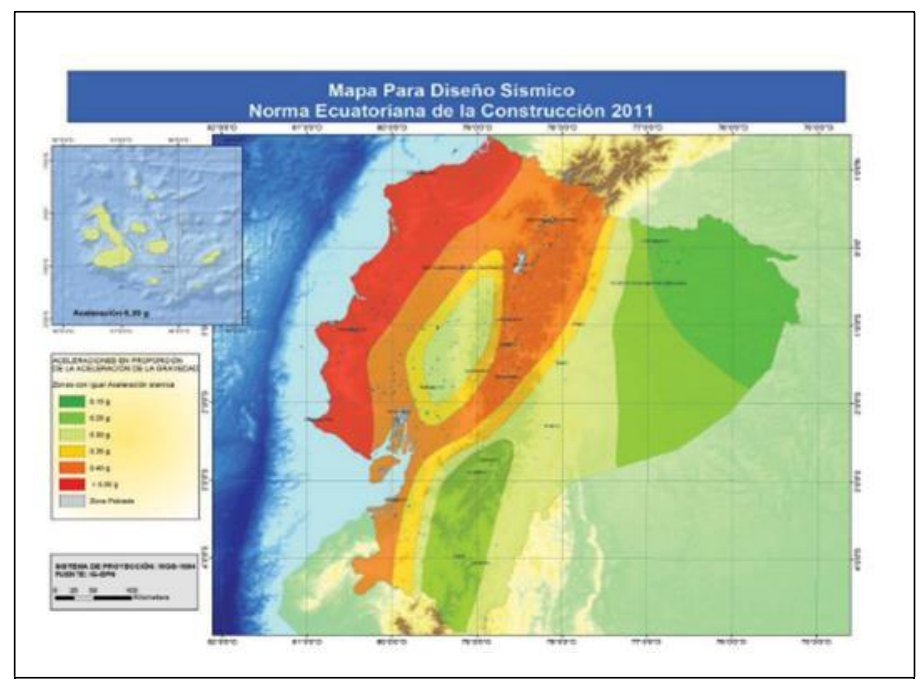

Figura $N^{\circ}$ 17.- Zonas sísmicas del Ecuador [8] 


\begin{tabular}{|l|l|l|l|l|l|l|}
\hline Zona sismica & I & II & III & IV & V & VI \\
\hline Valor factor Z & 0.15 & 0.25 & 0.30 & 0.35 & 0.40 & $\geq 0.50$ \\
\hline $\begin{array}{l}\text { Caracterización del } \\
\text { peligro sísmico }\end{array}$ & Intermedia & Alta & Alta & Alta & Alta & Muy alta \\
\hline
\end{tabular}

\section{Tabla $N^{\circ}$ 3.- Valores de factor de aceleración sísmica [8]}

Se toma un valor superior de aceleración sísmica de 0.25 por tratarse de una estructura como un oleoducto que transporta un material contaminante e inflamable.

Con la información de riesgo sísmico, los parámetros geotécnicos de los ensayos tri -axiales de suelos y el perfil del levantamiento topográfico se realiza un análisis de estabilidad de los taludes identificados como más críticos mediante un modelado con programa especializado de elementos finitos.

La figura 18, muestran parte de los resultados obtenidos al realizar la simulación, en este caso para la abscisa $1+750$ de la variante [3].

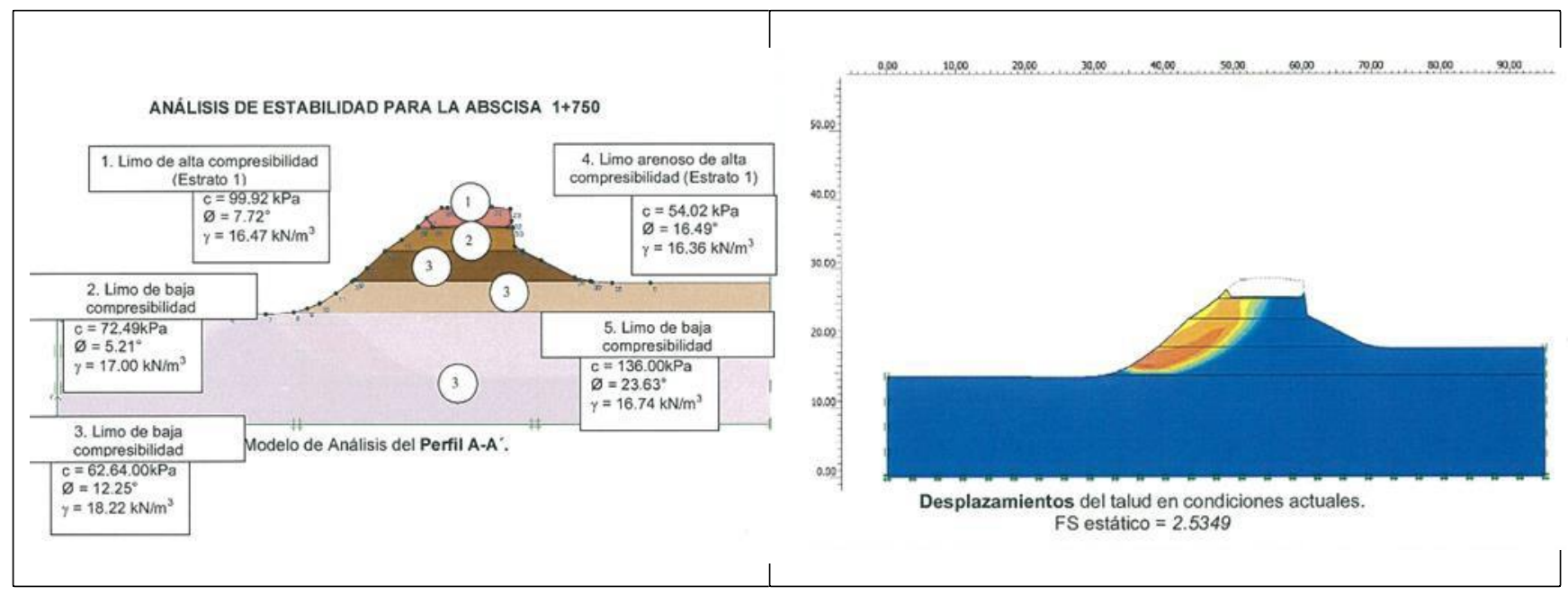

Figura $N^{\circ}$ 18.- Resultados de simulación de estabilidad de taludes [3]. 
Carlos R. Chávez-Romero; Rene A. Carrillo-Flores; Darwin R. Caina-Aysabucha; Marcelo P. Carrillo-Flores; Mariana G.

Los resultados finales se muestran en la tabla 4 para los 3 taludes considerados en riesgo de inestabilidad. La abscisa 0+000 es el inicio de la variante, aproximadamente en el kilómetro 19 del derecho de vía.

\begin{tabular}{|c|c|c|}
\hline Abscisa & $\begin{array}{l}\text { Factor de } \\
\text { seguridad } \\
\text { estático }\end{array}$ & $\begin{array}{l}\text { Factor de } \\
\text { seguridad } \\
\text { dinámico }\end{array}$ \\
\hline $1+690$ & 1.980 & 1.054 \\
\hline $1+160$ & 2.086 & 1.387 \\
\hline $1+750$ & 2.535 & 1.889 \\
\hline
\end{tabular}

Tabla $N^{\circ}$ 4.- Factores de seguridad para taludes críticos. Autoría personal en base a la información de [3]

Los factores de seguridad estáticos muestran estabilidad para los taludes en estudio bajo condiciones estáticas; sin embargo, en el análisis para factores de seguridad pseudo-estáticos (dinámicos) se determinan valores en el límite $(\mathrm{FS}=1)$ los cuales podrían ser incluso menores al valor 1.0 si es que se incluyen las condiciones de saturación del suelo, es decir se pueden esperar deslizamientos bajo efectos de cargas sísmicas. Por tanto, es indispensable la construcción de obras de control de humedad del suelo.

Estos valores corresponden a factores de seguridad aceptables para la condición estática; sin embargo, se debe considerar la necesidad de garantizar la estabilidad del área a través de la construcción de obras de canalización de agua. 


\section{Evaluación de cantidades de obra.}

Con el levantamiento topográfico ejecutado, como parte del estudio de campo, se realiza el diseño vertical y horizontal del derecho de vía, garantizando de esta manera el aprovechamiento del suelo y una adecuada canalización de las aguas.

Las características de la variante planteada son las siguientes [3]:

Longitud del derecho de vía de la variante: $2972.37 \mathrm{~m}$

- Área de Desbroce y Limpieza: $\quad 3.238 \mathrm{Ha}$

- Movimiento de tierras:

- Volumen de Corte: $\quad 24.494 .21 \mathrm{~m} 3$

- Volumen de Relleno $\quad 536.78 \mathrm{m3}$

- Corte y relleno en zanja: $\quad 4.527 .00 \mathrm{~m} 3$

- Longitud de la tubería desarrollada: $\quad 3.017 .86 \mathrm{~m}$

- Longitud de compra de derecho de vía: 3000 m x 30 m de ancho = 90000 m2.

En base a las cantidades de obra se estima un presupuesto de construcción del derecho de vía y de la tubería de la variante. Los detalles se presentan en la tabla 5.

El espesor de la tubería a ser utilizada en la variante se calcula usando la fórmula clásica de los cilindros de paredes delgadas más los factores de seguridad correspondientes [9].

$$
\mathrm{t}=\frac{\mathrm{PD}}{2 \mathrm{~S} \mathrm{FET}}
$$

Dónde:

P es la presión de diseño, en el caso presente 1400 psi;

D es el diámetro externo en pulgadas, 12.75; 
Carlos R. Chávez-Romero; Rene A. Carrillo-Flores; Darwin R. Caina-Aysabucha; Marcelo P. Carrillo-Flores; Mariana G.

S es la resistencia mínima de fluencia, 52000 psi

F es el factor de seguridad que se toma 0.6 para el presente caso ya que la Compañía considera el sector como de clase II de acuerdo a la clasificación de ASME B31.8 (840.2.2);

E es el factor de eficiencia de junta que es de 1 en este caso para tubería sin costura de acuerdo a la tabla 841.1.7-1 de ASME B31.8;

T es el factor de temperatura, mismo que es de 1 para tuberías que trabajan a menos de $250^{\circ} \mathrm{F}$ de acuerdo a la tabla

\subsection{8-1 de ASME B31.8; [5]}

Considerando los valores anteriores y un espesor de corrosión de 2 millas por año, para un valor de diseño de 20 años de vida útil, se tiene que el espesor calculado es de 0.326 pulgadas, lo que en espesores comerciales disponibles es de 0.330 pulgadas.

Esta información permite realizar un alcance licitatorio bien determinado que evita re-ajustes por cantidades de obra y trabajos no contemplados, mismos que se traducen en costos adicionales. El error estimado en el costo es de $\pm 10 \%$ que es la consideración que se hace de acuerdo al programa del Project Management Institute cuando se cuenta con ingeniería de detalle y experiencias previas de costos en otros proyectos.

La información que maneja la empresa que administra el oleoducto considera un costo mínimo de USD 1200000 por derrame de magnitud reducida, como lo que se han producido en esta zona desde 2006. Este costo incluye limpieza del área, reemplazo de sección afectada, indemnizaciones. A esto se deben sumar otros costos no fácilmente cuantificables como la 
afectación de imagen que en casos de las compañías petroleras es muy sensible. Puede evidenciarse que vale la pena construir la variante tanto desde el punto de vista de reducción de riesgo como el económico.

\section{Construcción de la variante}

La construcción de la variante no representa técnicamente una dificultad mayor. Como en la mayoría de los proyectos que se realizan en Ecuador, la ruta crítica la constituyen los materiales que en su gran mayoría son importados y deben ser transportados por vía marítima.

El objetivo es realizar la construcción en el menor tiempo posible de manera que se minimicen las posibilidades de un nuevo evento en el área. El tiempo necesario para la llegada de la tubería es de 6 meses.

En la figura 19 se aprecia la ruta de la variante implantada, alejándose de la vía existente y la sección del derecho de vía determinada como inestable. 
Carlos R. Chávez-Romero; Rene A. Carrillo-Flores; Darwin R. Caina-Aysabucha; Marcelo P. Carrillo-Flores; Mariana G.

\section{Calderón-Defaz}

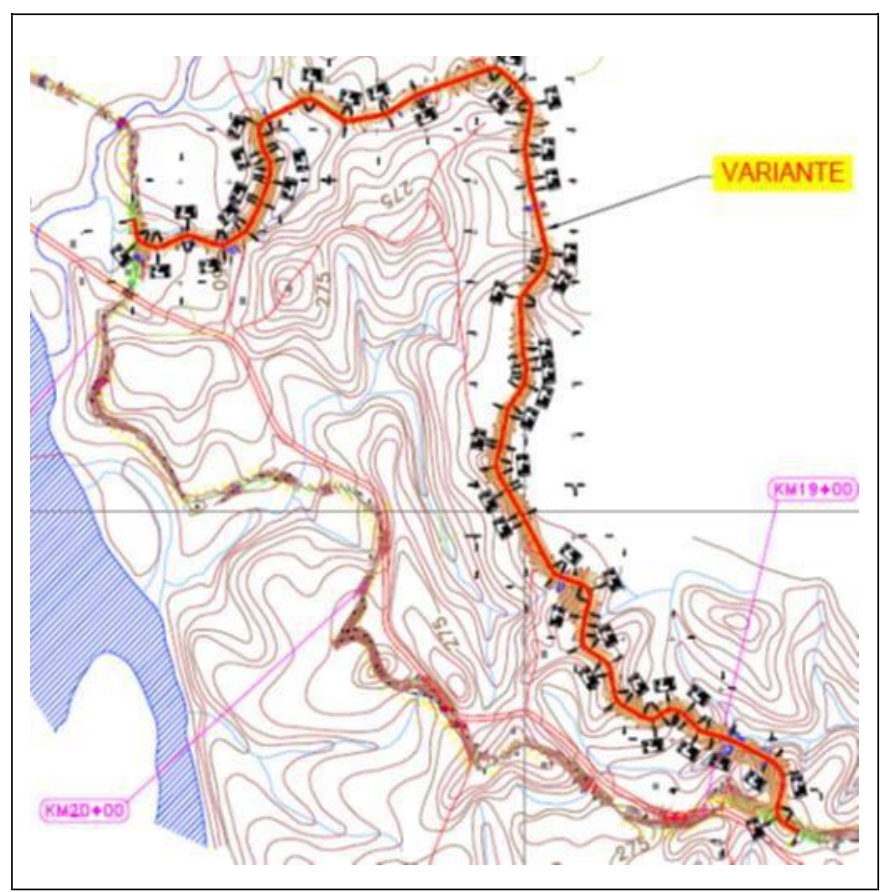

Figura $N^{\circ}$ 19.- Variante implantada. Autoría Personal.

\begin{tabular}{|c|c|c|c|c|c|}
\hline No. & Detalle & Unidad & Cantidad & $\begin{array}{l}\text { Costo } \\
\text { unitario }\end{array}$ & Costo total \\
\hline 1 & $\begin{array}{l}\text { Construcción } \\
\text { del derecho de } \\
\text { vía }\end{array}$ & $\mathrm{m} 3$ & 25000 & 9.00 & $\begin{array}{r}\$ \\
225.000 .00\end{array}$ \\
\hline 2 & $\begin{array}{l}\text { Compra de } \\
\text { tubería 12" API } \\
\text { 5L X52 FBE } \\
\text { DRD }(+10 \%) .\end{array}$ & $\mathrm{m}$ & 3.300 .00 & $\$ 143.00$ & $\$ 471.900 .00$ \\
\hline 3 & $\begin{array}{l}\text { Compra de } \\
\text { terrenos para el } \\
\text { DDV con un } \\
\text { ancho de } 30 \\
\text { metros }\end{array}$ & $\mathrm{m} 2$ & 90.000 .00 & $\$ \quad 0.50$ & $\$ 45.000 .00$ \\
\hline 4 & $\begin{array}{l}\text { Compensaciones } \\
\text { a la comunidad }\end{array}$ & Global & 1.00 & $\begin{array}{l}\$ \\
50.000 .00\end{array}$ & $\$ 50.000 .00$ \\
\hline 5 & $\begin{array}{l}\text { Construcción de } \\
\text { variante }\end{array}$ & $\mathrm{Km}$ & 3.00 & $\begin{array}{l}\$ \\
200.000 .00\end{array}$ & $\begin{array}{r}\$ \\
600.000 .00\end{array}$ \\
\hline 6 & $\begin{array}{l}\text { Retiro de tubería } \\
\text { existente a ser } \\
\text { abandonada }\end{array}$ & $\mathrm{m}$ & 3.000 .00 & $\$ 55.00$ & $\$ 165.000 .00$ \\
\hline & & & \multicolumn{2}{|l|}{ Costo total } & $\$ 1.556 .900 .00$ \\
\hline
\end{tabular}

Tabla $N^{\circ}$ 5.- Presupuesto estimado para variante. Autoría personal. 


\section{Plan de mantenimiento.}

El plan de mantenimiento para el oleoducto en general y para el área de la variante en particular está diseñado en base a un análisis de riesgos de manera que los recursos económicos y humanos se focalicen en las áreas más críticas.

De primera mano se conocen 3 puntos donde los taludes presentan un riesgo de inestabilidad. En estas tres zonas se instalarán sistemas de monitoreo superficiales de movimientos mediante mojones, realizándose tomas de coordenadas e inspecciones de campo cada 3 meses. En caso de que estas tomas o las inspecciones de campo muestren evidencias de movimientos del terreno, se deberán realizar inspecciones de campo para evaluar la necesidad de ejecutar obras complementarias adicionales. Si las inspecciones y mediciones mediante mojones indican que no existe evidencia de movimiento, se evaluará la frecuencia de inspección a fin de extenderla a un intervalo más conveniente.

Los recorridos regulares de inspección en sitio se iniciarán en la variante con una frecuencia de 3 meses y en base a los resultados se revisará la misma. El mantenimiento de cunetas laterales y de coronación, así como las novedades en sectores adyacentes que puedan tener consecuencias en el derecho de vía, deberán ser registrados y reportados a las instancias correspondientes.

El programa de mantenimiento del derecho de vía deberá ceñirse a lo señalado en el "Procedimiento de Administración de Derechos de Vía” aprobado por la compañía dueña de oleoducto.

\section{Conclusiones.}

Toda obra ejecutada por el hombre altera el entorno. Esto debe ser considerado ya que la naturaleza buscará el equilibrio, lo que puede acarrear consecuencias no deseadas. 
Carlos R. Chávez-Romero; Rene A. Carrillo-Flores; Darwin R. Caina-Aysabucha; Marcelo P. Carrillo-Flores; Mariana G.

Se establecen con claridad las causas de los eventos producidos desde el año 2006, mismas que se deben básicamente a inestabilidad de suelos.

La solución planteada permite reducir el riesgo de nuevos eventos independientemente terceras partes.

La falta de evaluación de las condiciones geotécnicas del derecho de vía en la etapa de diseño, produce graves problemas durante la operación del oleoducto.

Pese a que Ecuador es un país con historial sísmico y cordilleras con diferentes clases de suelos, la investigación referente a riesgos de oleoductos por consideraciones geotécnicas es incipiente.

Como lecciones aprendidas se establecen las siguientes necesidades en el diseño de ductos:

Estudios geológicos y geotécnicos de la ruta.

Recorrido de derecho de vía por equipo multidisciplinario de profesionales de manera que puedan ubicarse zonas de riesgo como asentamientos, deslizamientos antiguos, zonas inestables.

Conservar en lo posible los espesores a lo largo del ducto de manera que se puedan realizar inspecciones de deformaciones mediante placas calibradoras.

La elección de rutas para ductos en general debe considerar la influencia que estructuras naturales o creadas por el hombre puedan tener sobre la integridad de los mismos. El deterioro con el paso del tiempo de esas estructuras debe ser considerado, así como la posibilidad de que reciban un mantenimiento adecuado. 
Se hace evidente la falta de control de los entes reguladores en la ingeniería básica y de detalle de los oleoductos.

En base a los resultados de este estudio, otras secciones del derecho de vía del oleoducto se encuentran bajo análisis, habiéndose encontrado hasta el momento otra área con problemas similares.

\section{Bibliografía.}

[1] ASME DiGETAL COLlECTION, Development of a Pipeline Wrinkle Material Ultimate Limit State: Full Scale Modeling. Presented at 2008 7th pipeline International Pipeline Conference, Calgary Alberta. Available:http:// asme.org/proceeding.aspx?articleid=1640712.

proceedings.asmedigitalcollection.

[2] DS Consultores, Estudios Geotécnicos para Determinar la Estabilidad de Tubería en kilómetros 1920 de Oleoducto, Junio 2014. Información confidencial de la operadora del ducto.

[3] DS Consultores, Estudio de Mecánica de Suelos de la Variante de Derecho de Vía de Oleoducto, Febrero 2015. Información confidencial de la operadora del ducto.

[4] Jornal of the Brazilian Society of Mechanical Sciences and Engineering: Buckling Analysis of High Temperature Pressurized Pipelines with Soil - Structure Interaction, Available: http://www.scielo.br/scielo.php?pid=S1678-58782003000200008\&script=sci_arttect\&tlng=pt

[5] Gestión de Integridad de Sistemas de Gasoductos, Código ASME B31.8S, 2010.

[6] Managing System Integrity for Hazardous Liquid Pipelines, API Standard 1160, 2001

[7] Manual del Ingeniero Civil, 3rd ed, Frederick Merritt, 1992

[8] Norma Ecuatoriana de la Construcción, Ministerio de Desarrollo Urbano y Vivienda, 2014

[9] Pipeline Transportation Systems for Liquids and Slurries, ASME Code for Pressure Piping B31.2, 2012.

[10] PROCESS Corp., MA. Intranets: Internet technologies deployed behind the firewall for corporate productivity. Presented at

INET96 Annual Meeting. [Online]. Available: http://home.process.com/Intranets/wp2.htp

[11] Reporte interno de historial de fallas de oleoducto. Información confidencial de la operadora del ducto. 10 
Carlos R. Chávez-Romero; Rene A. Carrillo-Flores; Darwin R. Caina-Aysabucha; Marcelo P. Carrillo-Flores; Mariana G.

[12] Reporte interno de inspección de falla de oleoducto, 2014. Información confidencial de la operadora del ducto.

[13] Rosen, Final Report Rocombo Inspection Service, February 2015. Información confidencial de la operadora del ducto.

[14] U.S. National Center for Earthquake Engineering Research, Buffalo, N.Y, Failure Criterion For Buried Pipe Subject to Longitudinal Permanent Ground Deformation. Available: http://www.cridlac.org/digitalization/pdf/eng/doc7488.html 